\title{
Early-stage laryngeal squamous cell carcinoma of the epiglottis treated by endoscopic submucosal dissection
}

Recent efforts by gastrointestinal endoscopists have enabled detection of laryngeal and pharyngeal cancer at an early stage, especially in Japan [1-3]. In addition, endoscopic mucosal resection (EMR) and endoscopic submucosal dissection (ESD) [4] have recently been used for treatment of hypopharyngeal cancer at an early stage. In this report, we describe for the first time a case of earlystage laryngeal carcinoma that was successfully treated by ESD.

A 61-year-old man was admitted to Hokkaido University Hospital because of a laryngeal cancer, which was found by follow-up endoscopy after EMR of esophageal cancer. The patient had a history of heavy smoking and alcohol consumption. On endoscopic examination, a round, flatelevated lesion measuring $1.5 \times 1.0 \mathrm{~cm}$ was noted on the front right part of the epiglottis ( Fig. 1). The surface was reddish and granular without any ulcerations. A whole-body CT scan demonstrated no lymphadenopathy. ESD was performed with the patient under general anesthesia. Submucosal dissection was done with a needle knife, and was performed successfully without complications ( $\bullet$ Fig. 2 a). Histopathologic examination of the resected specimen showed that squamous cell carcinoma had very slightly invaded the subepithelial layer ( $\bullet$ Fig. $\mathbf{2}$ b, c). The margins of the resected specimen were free histologically. There was no invasion into veins or lymph vessels. There has been no recurrence during a follow-up period of 9 months after ESD. In this case, by using the ESD technique, an en-bloc resected specimen and accurate histological evaluation were obtained without the occurrence of any complication such as injury to the epiglottis. Gastrointestinal endoscopy can now provide images of higher quality than those obtained by laryngoscopy. Gastrointestinal endoscopists should strive to find supraglottic cancer at an early stage in the laryngeal area for pa-
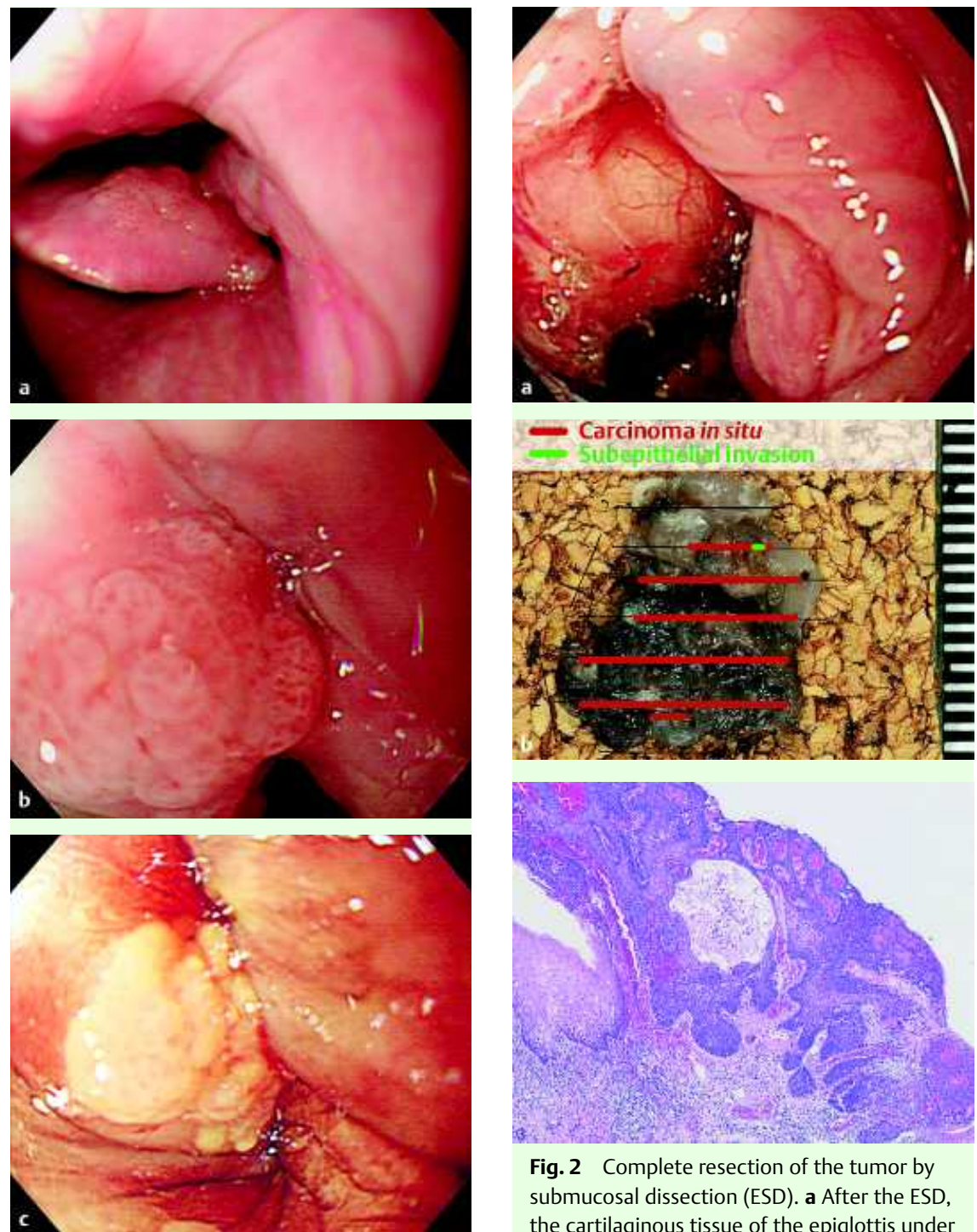

Fig. 1 Endoscopic view showing the right suprahyoid epiglottis of larynx. a Distant view showing granular elevated erythematous lesion. b Close-up view showed an area of reddish dots on the lesion. c Endoscopic iodine staining showed an unstained area that conformed with the lesion.

tients in the high-risk group such as heavy drinkers and heavy smokers [3], and of course it is necessary to cooperate with the otolaryngologist to treat.
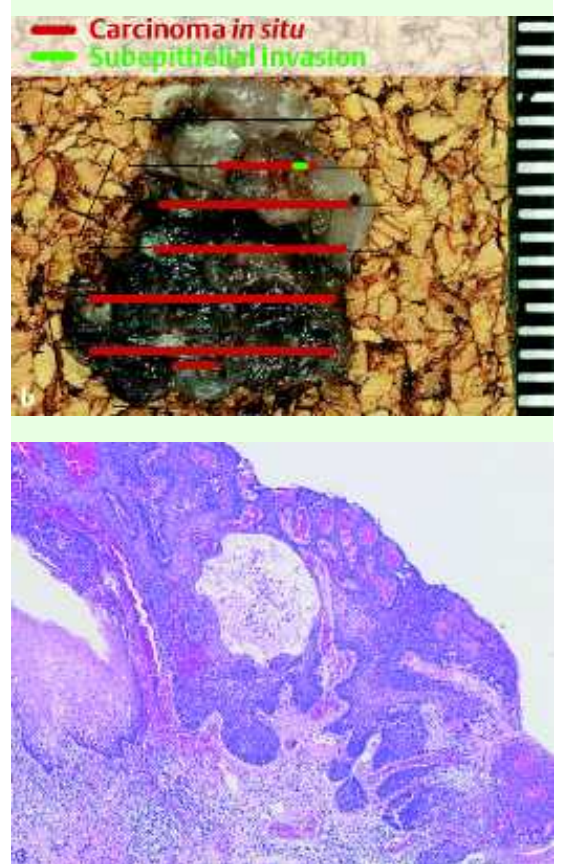

Fig. 2 Complete resection of the tumor by submucosal dissection (ESD). a After the ESD, the cartilaginous tissue of the epiglottis under tumor was visible through the transparent subepithelial layer. $\mathbf{b}$ Macroscopic image of the resected specimen. $\mathbf{c}$ Photomicrograph of the ESD specimen showing the lesion elevated from the epithelial mucosa and very slight invasion into the subepithelial layer (hematoxylin \& eosin; original magnification $\times 200$ ).

Endoscopy_UCTN_Code_TTT_1AO_2AG Endoscopy_UCTN_Code_TTT_1AO_2AD 
T. Yoshida ${ }^{1}$, Y. Shimizu ${ }^{2}$, J. Hirota ${ }^{1}$, M. Nakagawa' ${ }^{1}$, S. Ono ${ }^{2}$, N. Oridate ${ }^{3}$, M. Kato ${ }^{2}$, M. Asaka ${ }^{1}$

1 Department of Gastroenterology, Hokkaido University Graduate School of Medicine, Sapporo, Hokkaido, Japan

2 Division of Endoscopy, Hokkaido University Hospital, Sapporo, Hokkaido, Japan

3 Department of Otolaryngology, Hokkaido University Graduate School of Medicine, Sapporo, Hokkaido, Japan

\section{References}

1 Shimizu Y, Tsukagoshi H, Fujita M et al. Head and neck cancer arising after endoscopic mucosal resection for squamous cell carcinoma of the esophagus. Endoscopy 2003 35: $322-326$

2 Nagai K, Kawada K, Nishikage T et al. Endoscopic treatment for superficial hypopharyngeal carcinoma [in Japanese with English abstract]. Stomach Intestine 2003; 38 : $331-338$

3 Muto M, Nakane M, Katada $C$ et al. Squamous cell carcinoma in situ at oropharyngeal and hypopharyngeal mucosal sites. Cancer 2004; 101: 1375-1381

4 Shimizu Y, Yamamoto J, Kato $M$ et al. Endoscopic submucosal dissection for treatment of early stage hypopharyngeal carcinoma Gastrointest Endosc 2006; 64: 255-259
Bibliography

DOI 10.1055/s-2008-1077443

Endoscopy 2008; 40: E204-E205

(C) Georg Thieme Verlag KG Stuttgart · New York . ISSN 0013-726X

\section{Corresponding author}

\section{T. Yoshida, MD}

Department of Gastroenterology

Hokkaido University Graduate School of Medicine

Division of Endoscopy

Hokkaido University Hospital

Nshi-7, Kita-15, Kita-ku, Sapporo

Hokkaido 060-8638

Japan

Fax: +81-11-7067867

peugeot307ccs16@yahoo.co.jp 